\title{
Uso por Alumnos de Bachillerato de los Fenómenos Aproximación Simple Intuitiva y Retroalimentación en Sucesiones
}

\author{
High-School Students' Use of Intuitive Simple Approximation and \\ Feedback Phenomena in Sequences
}

\author{
Francisco-Javier Claros Mellado* \\ Teresa Sánchez Compaña ${ }^{* *}$ \\ Moisés Coriat Benarroch ${ }^{* * *}$
}

\begin{abstract}
Resumen
Relatamos los pasos seguidos para elaborar un cuestionario destinado a observar los fenómenos de aproximación simple intuitiva y de retroalimentación en las respuestas que dan alumnos de bachillerato ${ }^{* * * * *}$ a cuestiones sobre el límite finito de una sucesión. Presentamos el marco didáctico y metodológico de nuestras investigaciones. En el trabajo de campo descrito, participaron 143 alumnos de tres institutos diferentes de Madrid y su periferia. Incluimos resultados agregados, las categorías usadas para clasificar las respuestas, el uso que los alumnos hacen de los dos fenómenos mencionados y algunas dificultades que nuestras preguntas han planteado a los alumnos.
\end{abstract}

Palabras Clave: Límite Finito de Sucesiones. Fenomenología. Sistemas de Representación. Libros de Texto de Bachillerato. Diseño de una Encuesta.

\begin{abstract}
* Doctor en Didáctica de la Matemática por la Universidad de Granada (UGR). Profesor asociado de la Universidad Complutense de Madrid (UCM), Madrid, España. Dirección Postal: Av. Rector Royo Villanova s/n. Fac. de Educación. Dep. de Didáctica de las Matemáticas. Despacho 351728040 Madrid. E-mail: fclaros@ucm.es.

** Doctor en Didáctica de la Matemática por la Universidad de Granada (UGR). Profesora asociada de la Universidad de Málaga (UMA), Málaga, España. Dirección Postal: Bulevar de Luis Pasteur, Campus de Teatinos. Fa. De Educación. Dep. de Didáctica de las Matemáticas. Despacho 2.5229010 Málaga. E-mail: teresasanchez@uma.es.

${ }^{* * * *}$ Doctor en Ciencias Físicas, por Paris VI. Profesor Jubilado de la Universidad de Granada (UGR), Granada, España. Dirección Postal: Facultad de Educación, campus de la Cartuja s/n 18071, Granada. Insertar. E-mail: mcoriat@ugr.es

El Bachillerato (B.) designa en España, desde 1990, la Educación Secundaria No Obligatoria. Se realiza en dos cursos que, normalmente, corresponden a las edades 16-17 y 17-18 años. Los centros en los que se estudia se denominan Institutos. Existen varias modalidades: B. de Ciencias de la Naturaleza y de la Salud, B. Tecnológico y B. de Ciencias Sociales. Desde 1990 han cambiado dos veces los currículos de matemáticas. Éstos son aproximadamente idénticos en las dos primeras modalidades. Se suele traducir en inglés como High.School. En este artículo se mencionan libros de texto de Bachillerato que abarcan un período de 70 años; el lector debe estar advertido que el B. en España ha experimentado importantes cambios estructurales en dicho lapso.
\end{abstract}


We report the steps followed to set up an instrument oriented to detect two phenomena (intuitive simple approximation and feedback) in the answers given by high school students to questions concerning the finite limit of a sequence. We present our research's didactical and methodological frames. Along the field work described, we could collect answers from 143 students in three different high schools, in Madrid and on the outskirts. We include several global results, the criteria used to classify answers, the use that students make of the mentioned phenomena, and some difficulties that the students found when faced with our questions.

Keywords: Finite limit of a Sequence. Phenomenology. Representation Systems. High-School Level Textbooks. Design of a Survey.

\section{Introducción}

Presentamos algunos avances de ideas extraídas de una tesis doctoral (CLAROS, 2010) relativas al límite finito de sucesiones y a las respuestas de alumnos de bachillerato a cuestiones sobre dicho límite. Allí introdujimos dos fenómenos (en el sentido de FREUDENTHAL, 1983), uno de aproximación intuitiva y otro de retroalimentación, que resumimos más abajo (apartado 3). Dichos fenómenos deben ser observables en los libros de textos (de los que elegimos una treintena) y en el comportamiento de los resolutores (alumnos de bachillerato).

Para remontar desde los libros de texto o los trabajos de los alumnos hasta los fenómenos propiamente dichos, se necesita filtrar los contenidos observados y eliminar, en lo posible, los estilos personales o la contaminación que el propio investigador puede generar.

Este artículo se estructura en dos partes. En la primera (apartados 2 y 3), exponemos el marco didáctico y matemático, mientras que, en la segunda (apartados 4 a 7), exponemos el método seguido, el trabajo de campo propiamente dicho y los resultados globales obtenidos.

En el siguiente apartado describimos investigaciones en Educación Matemática que consideramos relevantes ya que conforman el marco teórico de nuestro trabajo de investigación desarrollado desde hace años. El tercer apartado, de orientación matemática, resume los fenómenos de aproximación intuitiva y retroalimentación que queremos reconocer en las respuestas de los alumnos. Estos fenómenos han sido presentados en diferentes publicaciones, como Claros (2010) o Claros, Sánchez y Coriat (2006).

En el cuarto apartado exponemos las etapas que permitieron elaborar un instrumento de recogida de información y resolver dificultades encontradas. En primer lugar, elaboramos un cuestionario y lo sometimos al juicio de expertos, profesores de instituto, cuyas aportaciones permitieron valorar la pertinencia o relevancia de las preguntas y ayudaron a construir un cuestionario piloto. En segundo lugar, el cuestionario piloto se administró a un grupo de alumnos de $2^{\circ}$ de bachillerato, del instituto José Hierro (Getafe, Madrid). En la 
corrección de las respuestas al cuestionario piloto, vimos la necesidad de refinar las categorías de análisis de las respuestas que sirvieran para futuras actuaciones orientadas a reconocer los fenómenos en las producciones de los alumnos. Tras el estudio de las respuestas a dicho cuestionario piloto, estuvimos en condiciones de elaborar el cuestionario.

El quinto apartado se orienta a describir el proceso de administración del cuestionario. Los cuestionarios se corrigieron usando las categorías de análisis mencionadas, de manera que cada respuesta dada por un alumno a cada pregunta quedó asociada a una sola categoría.

El sexto apartado incluye, en primer lugar, un análisis descriptivo de los resultados y, en segundo lugar, una propuesta para relacionar las respuestas obtenidas y el uso de los fenómenos.

En el apartado séptimo realizamos un estudio comparativo entre los resultados obtenidos del cuestionario y los resultados obtenidos con una muestra de 30 libros de texto, publicados entre 1933 y 2005 (CLAROS, 2010).

El apartado octavo resume los principales resultados obtenidos y expone algunas expectativas.

Primera parte: marcos didáctico y matemático

\section{Antecedentes}

La enseñanza del límite es difícil. GUCLER (2010) describe dificultades relativas a los alumnos; considera que, tras la explicación a una clase del concepto de límite, se repiten dificultades que se han tenido que superar históricamente para formalizar el concepto.

Claros, Sánchez y Coriat (2006) señalaron dificultades relativas al límite finito de una sucesión y el límite finito de una función en un punto. Exponen que el límite finito de una sucesión organiza dos fenómenos cuya descripción remitimos al apartado 3.

La introducción de estos fenómenos en la enseñanza del límite de sucesiones pretende arrojar luz sobre las dificultades generadas por esta noción. De hecho, el marco teórico que se manejó en la investigación de Claros (2010) puso de manifiesto la necesidad de conjugar fenomenología, pensamiento matemático avanzado y representaciones.

Cuando hablamos de fenomenología lo hacemos en el sentido de Freudenthal (1983, p.28):

Phenomenology of a mathematical concept, a mathematical structure, or a mathematical idea means, in my terminology, describing this noumenon in relation to the phenomena of which it is the means of organising, indicating which phenomena it is created to organise, and to which it can be extended, how it acts upon these phenomena as a means of organising, and with what power over these phenomena it endows us. 
Freudenthal afirma que noumeno es objeto del pensamiento, mientras que fenómeno es algo de lo que tenemos experiencia. Para este autor los conceptos matemáticos no caen fuera del campo de nuestra experiencia, ni están en un mundo distinto del mundo de los fenómenos que organizan. Un concepto matemático que es el medio de organización de un fenómeno (o más de uno), pasa a formar parte de un campo de fenómenos que son organizados por un concepto matemático nuevo.

En el congreso de 1985 de Psycology of Mathematics Education, se creó un grupo de trabajo para estudiar la naturaleza del pensamiento matemático avanzado (PMA); básicamente, pretendía profundizar en los procesos cognitivos de enseñanza y aprendizaje de temas relacionados con el cálculo infinitesimal (DREYFUS, 1990; TALL, 1991). El límite es un concepto que se sitúa dentro del cálculo infinitesimal, la derivada y la integral (para muchos, también la continuidad) se definen a partir de él. Además, el concepto de límite se considera abstracto y se suele situar en el ámbito de las llamadas matemáticas superiores. Autores como Tall (1991) y CORNU (1991) concuerdan en situar el límite dentro del PMA: Tall (1991) señala que el concepto de límite se sitúa dentro del PMA por los procesos cognitivos que son necesarios para su manejo; por su parte Cornu (1991) también lo hace, al observar que el límite es una pieza fundamental en la teoría de las aproximaciones, continuidad, derivabilidad e integración.

EDWARDS, DUBINSKY y MCDONALD (2005) indican, sin embargo, que el límite estará situado en el pensamiento matemático elemental (PME) o avanzado dependiendo del trabajo que se realice con él. Si solamente se trabaja cálculo de límites, no estaríamos hablando de un concepto que requiera un pensamiento matemático avanzado para realizar esta operación.

Teniendo en cuenta los fenómenos de aproximación intuitiva y retroalimentación el concepto de límite finito de una sucesión está dentro del PMA si los alumnos reconocen y emplean los dos fenómenos de manera conjunta y coordinada en sus justificaciones. En cambio, si los alumnos emplean únicamente la aproximación intuitiva, el límite finito de una sucesión lo incluimos en el PME. El Cuadro 1 sintetiza las relaciones que acabamos de exponer.

\begin{tabular}{|c|c|c|}
\hline FENÓMENOS (ver Apartado 3) & En PME & En PMA \\
\hline De aproximación intuitiva & Se usa & Se usan en el orden que se \\
indica aquí.
\end{tabular}

Cuadro 1 - Fenómenos y pensamiento matemático avanzado 
Es necesario considerar el papel de las representaciones en el desarrollo de las matemáticas y su aprendizaje por parte de los alumnos. Siguiendo a JANVIER (1987), entendemos que se usan cuatro sistemas de representación en la enseñanza del límite: simbólico, gráfico, tabular y verbal. Estos sistemas de representación fueron denominados por BLAZQUEZ Y ORTEGA (2000) verbal, gráfico, simbólico y numérico. Hemos preferido el término tabular al término numérico. Teniendo en cuenta esto, hablamos de fenómenos organizados por el límite finito de una sucesión en diferentes sistemas de representación.

Los fenómenos de aproximación simple intuitiva (a.s.i) y retroalimentación o idavuelta en sucesiones (i.v.s) se presentaron en Claros (2010) y Claros, Sánchez y Coriat (2006; 2013) y fueron observados en una muestra de 30 libros de texto que se presentó en Claros (2010) y en Claros, Sánchez y Coriat (sometido para publicación). Los fenómeno a.s.i e i.v.s se pueden presentar en los sistemas de representación: verbal, gráfico, simbólico y tabular y en los formato ejemplo y definición. En Claros (2010), se señaló cómo la mayor o menor frecuencia de los diferentes fenómenos en los libros de texto está estrechamente relacionada con el periodo educativo en el que se ha elaborado el libro de texto.

Queremos observar si los alumnos de $1^{\circ}$ y $2^{\circ}$ de bachillerato usan los fenómenos de aproximación intuitiva y de retroalimentación cuando responden a un cuestionario sobre el límite finito de una sucesión y determinar la frecuencia con que lo hacen. En las respuestas de los alumnos tendremos en cuenta el sistema de representación y el formato (ejemplos o definiciones) usados para presentar dichos fenómenos.

\section{Fenómenos de aproximación simple intuitiva y retroalimentación o ida-vuelta}

Nuestro estudio del límite de sucesiones partió del siguiente enunciado:

Definición: Sea $x_{n}$ una sucesión en $R$, decimos que $x_{n}$ converge a un número real $x$ (o tiene como límite el real $x$ y escribimos $\lim x_{n}=x$ ) si para cada $\varepsilon>0$, existe un número natural $N$ tal que si $n>N$ se cumple que $\left|x_{n}-x\right|<\varepsilon$ (SPIVAK, 1991, p. 615).

El límite finito de una sucesión organiza dos fenómenos cuyo contenido resumimos a continuación. Véase Claros, Sánchez y Coriat (2006), Claros (2010) y Claros, Sánchez y Coriat (2013)

\subsection{Fenómeno de aproximación simple intuitiva (a.s.i)}


Cuando observamos una sucesión de números reales cuyo límite está correctamente declarado, notamos que los valores que toma se van acercando más y más a ese límite. Éste es el primer fenómeno que observamos en las sucesiones que tienen límite, también llamadas convergentes, y lo denominamos fenómeno de aproximación simple intuitiva (a.s.i).

La aproximación simple intuitiva remite a los valores que van tomando los términos de una sucesión de números reales con límite real.

El fenómeno de aproximación simple intuitiva permite obtener un candidato a límite o comprobar, grosso modo, que el candidato que nos proponen parece adecuado. No garantiza que el candidato seleccionado sea el límite de la sucesión presentada, porque la idea de acercarse cada vez más a un valor no ha quedado bien establecida con este fenómeno.

\subsection{Fenómeno de retroalimentación o ida-vuelta en sucesiones (i.v.s)}

La seguridad de que un candidato a límite es el límite de la sucesión presentada se consigue a través del fenómeno que llamamos retroalimentación o fenómeno de ida y vuelta en sucesiones (i.v.s); recibe este nombre porque controla dos procesos para establecer o descartar, sin lugar a dudas, el acercamiento indefinido de los valores de la sucesión a un candidato a límite; la observación conjunta y sucesiva de estos dos procesos da lugar a lo que denominamos fenómeno de retroalimentación o de ida-vuelta en sucesiones.

Desde una perspectiva métrica, que corresponde a la definición dada al comienzo de este apartado, la retroalimentación exige construir una función $\varepsilon$-n para sucesiones. Dicho en términos coloquiales y gráficos: una vez establecido el entorno en el límite con $\varepsilon$ dado determinamos si existe un correspondiente $n$ asociado (es decir, vamos-ida- desde algún lugar situado en el entorno del límite hacia la variable natural); hecho esto, volvemos - vuelta - al entorno del límite para comprobar que las imágenes así obtenidas pertenecen al entorno considerado.

Con el apoyo de la propia sucesión de referencia, la definición formal de límite finito de una sucesión induce la construcción simbólica de una función epsilon-n, o en su defecto la demostración de su existencia, la cual sirve para establecer una propiedad de la sucesión dada.

Segunda parte: Método, trabajo de campo y resultados

\section{Elaboración del instrumento: cuestionario y categorías}


El primer objetivo es el de detectar los fenómenos de aproximación intuitiva y retroalimentación en respuestas de alumnos de bachillerato a un cuestionario escrito. Para la construcción del instrumento, hemos seguido tres etapas.

Primera etapa. Elaboración de un Cuestionario Inicial y revisión por expertos: profesores de institutos.

Segunda etapa. (A) Analizar las sugerencias de los expertos. Enunciar las primeras categorías de análisis. (B) Elaborar una Cuestionario Piloto y administrar esta prueba con un grupo de ensayo. (C) Estudiar las respuestas de los alumnos. Tomar de decisiones sobre el instrumento.

Tercera etapa. Redacción definitiva del instrumento: Cuestionario y categorías de análisis asociadas.

\subsection{Primera Etapa}

En el periodo 1990-2005, en los libros de texto se daba una mayor frecuencia, en la definición de límite, del fenómeno de aproximación simple intuitiva (a.s.i) en detrimento del fenómeno de retroalimentación o ida-vuelta en sucesiones (i.v.s), (CLAROS, 2010; CLAROS; SÁNCHEZ; CORIAT, 2013). Por ello, una primera decisión consistió en estudiar, en las producciones de los alumnos, las diferentes presentaciones del fenómeno de aproximación simple intuitiva (a.s.i), de las que elegimos las representaciones verbal, gráfica o tabular en formato de ejemplos. Descartamos la representación simbólica porque no la observamos en los libros, así como el formato definición.

El cuestionario inicial incluía 12 preguntas y prestaba atención a las siguientes variables: (1) Se distinguió entre sucesión creciente y sucesión decreciente, por conjeturar que el carácter creciente o decreciente de una sucesión influiría en las respuestas obtenidas. (2) Se distinguió entre enunciado matemático y enunciado de la vida cotidiana, por conjeturar que los enunciados del último tipo ayudan a los alumnos a ver la aplicación de los conceptos matemáticos y esto puede hacer que se impliquen más en sus respuestas. (3) Se emplearon los sistemas de representación verbal, gráfico y tabular. (4) Se usaron sucesiones convergentes con límite finito, excluyendo situaciones atípicas, como las llamadas sucesiones dobles.

El Cuestionario Inicial, que se reproduce en el Anexo 1, lo sometimos a la consideración de 5 profesores del Departamento de Matemáticas del Instituto José Hierro (Getafe, Madrid) con experiencia en la enseñanza del límite de una sucesión. A la entrega del 
cuestionario se hizo la aclaración de que no se trataba de examinarlos a ellos, sino de ver si las preguntas del cuestionario son adecuadas para el aprendizaje del límite finito de una sucesión. Se pidió que anotaran libremente (es decir, sin guión previo elaborado por nosotros) sus sugerencias en el propio cuestionario. No recibieron explicación relativa a los fenómenos que deseamos observar, sólo se pidió la adecuación de las cuestiones enunciadas. El término adecuada es usado de manera intencionadamente genérica, para dejar un campo abierto a posibles críticas. Las principales recomendaciones de los expertos se indican a continuación, al justificar las decisiones que condujeron a la prueba piloto. Las respuestas recibidas se reproducen en el Anexo 2.

\subsection{Segunda etapa}

\subsubsection{Elaboración del Cuestionario Piloto}

Como consecuencia de las consideraciones de los expertos, tomamos las decisiones siguientes: (1) Supresión de los problemas de la vida real. Éstos ponen en juego connotaciones que no parecen necesarias para nuestro estudio. Por ejemplo: en un problema sobre economía (Anexo I, Pregunta 12) deberíamos manejar infinitas cifras decimales, lo cual carece de sentido precisamente en economía, donde no se consideran las cifras a partir de un cierto orden decimal. (2) Supresión de la distinción entre sucesión creciente y decreciente. Los profesores consideraron que las tareas en las que se distinguía en un mismo sistema de representación entre sucesión creciente y decreciente, no discriminaban sobre la definición de límite. (3) Eliminación, cuando se daba en alguna pregunta, del término general de la sucesión. Se evita, así, que los alumnos empleen teoremas sobre el cálculo de límites, en lugar de usar el sistema de representación propuesto en la pregunta. (4) Introducción liviana del fenómeno de retroalimentación o ida-vuelta en sucesiones (i.v.s) para buscar una cierta semejanza con lo que suele explicarse en algunas clases.

Teniendo en cuenta estos resultados, el Cuestionario Piloto constó de tres cuestiones, una para cada sistema de representación. La tercera pregunta incluyó dos partes, para tener en cuenta la representación verbal en ambos fenómenos.

Los objetivos de este cuestionario fueron los siguientes: (1) Detectar el fenómeno de aproximación simple intuitiva (a.s.i) en las respuestas de los alumnos. (2) Confirmar que los alumnos no manejan el fenómeno de retroalimentación o ida-vuelta en sucesiones (i.v.s). (3) 
Observar si los sistemas de representación usados en los enunciados de las preguntas (verbal, gráfico y tabular), tienen alguna influencia en las respuestas de los alumnos. (4) Detectar errores atribuibles a la elaboración del cuestionario.

Todas las cuestiones tuvieron la siguiente estructura: El profesor preguntaba a la clase si la sucesión presentada tenía límite. Un alumno ficticio daba una respuesta. Se pedía, a los sujetos, que dijeran si estaban de acuerdo con la respuesta del alumno ficticio y además que justificaran su propia respuesta. Todas las sucesiones presentadas tenían límite finito.

- En la primera pregunta se presentó gráficamente un ejemplo de sucesión cuyos valores se aproximaban a 1 y se preguntaba si dicha sucesión tenía límite 1 y que se justificara la respuesta. En este caso se usó en el enunciado el fenómeno a.s.i, la representación gráfica y el formato ejemplo.

- En la segunda pregunta se usó una tabla para describir los valores que iba tomando una sucesión. Estos valores, a medida que n crecía, se iban aproximando a 2. Se preguntaba si la sucesión tenía límite 2 y que se justificara la respuesta. En el enunciado de la pregunta se usó el fenómeno de aproximación simple intuitiva (a.s.i) en representación tabular y formato ejemplo.

- La pregunta número tres se compuso de dos partes. En la primera se usó el fenómeno de aproximación simple intuitiva (a.s.i) en el sistema de representación verbal y en el formato ejemplo para describir cómo los valores de la sucesión se aproximaban a 4. Se preguntaba si dicha sucesión tenía límite 4, y se pidió que se justificara la respuesta. En la segunda parte se usó el fenómeno de ida-vuelta en sucesiones en el enunciado, el sistema de representación verbal y el formato ejemplo para afirmar que la sucesión presentada tenía límite 4. Se pidió que el alumno expresara su respuesta a la pregunta y que la justificara. En este apartado se usó la palabra entorno para afirmar que 4 era el límite de la sucesión presentada. El Cuestionario Piloto se reproduce en el Anexo 3.

\subsubsection{Administración del Cuestionario Piloto}

Se administró a ocho alumnos de $2^{\circ}$ de Bachillerato de Ciencias Sociales del IES José Hierro (Getafe, Madrid). El criterio de selección de la muestra fue el siguiente: se eligieron aquellos alumnos que estaban trabajando en clase de manera individual. Con ello, quedaron fuera de la selección 7 alumnos que estaban sentados en grupos de tres y de dos; así, creíamos no alterar el ritmo de la clase: un grupo de 8 alumnos realizó un cuestionario, que no era un 
examen, porque no lo hacían todos, y los otros hicieron los ejercicios de clase propuestos por el profesor. Se pretendió crear ante la prueba un clima de naturalidad, como si se tratara de un ejercicio de clase. Hay que reseñar el interés que mostraron sobre las cuestiones presentadas.

Se pidió que rellenaran unos datos adicionales, necesarios para conocer su trayectoria escolar: nombre, curso, centro, nota de matemáticas en el curso anterior, edad, sexo, asignatura de matemáticas que cursan, y libro de texto que estaban manejando; también se preguntó si repetían curso o no.

\subsubsection{Estudio de las respuestas al Cuestionario Piloto}

Para el análisis de las respuestas se usaron las categorías que se muestran en el Cuadro 2. Éstas se construyeron partiendo de la experiencia de los investigadores (autores de este trabajo cuando, como docentes, enseñaban el límite de una sucesión.

\begin{tabular}{|c|l|}
\hline Categorías & \multicolumn{1}{c|}{ Descripción } \\
\hline C1 & Justifica empleando la respuesta dada en el enunciado \\
\hline C2 & Contesta erróneamente \\
\hline C3 & $\begin{array}{l}\text { Identifica la afirmación que aparece en el enunciado de la cuestión como la } \\
\text { definición de límite }\end{array}$ \\
\hline C4 & Responde bien y justifica su respuesta \\
\hline C5 & $\begin{array}{l}\text { Plantea la necesidad de conocer más valores de 'x' o ' } \mathrm{f}(\mathrm{x})^{\text {' }} \text {. Algunos alumnos } \\
\text { emplearon esta respuesta, en algunas de las preguntas del cuestionario. En este } \\
\text { caso los alumnos usaron la variable } \mathrm{x}, \text { para referirse a los valores } 1,2,3, \ldots . . \text { En } \\
\text { general suele usarse la variable "n" para sucesiones, en las que el dominio es N y } \\
\text { "x", para las funciones reales, en las que el dominio es R. }\end{array}$ \\
\hline C6 & No sabe / No contesta \\
\hline
\end{tabular}

Cuadro 2 - Categorías de análisis del Cuestionario Piloto

Los resultados y conclusiones se muestran en el Anexo 4. Los resultados más notables, que sirven como justificación para el desarrollo de la tercera etapa, son:

- Los alumnos usan en la mayoría de los casos la respuesta dada por el alumno ficticio como justificación de sus respuestas. Por ello la categoría C1 es la más frecuente (15 respuestas).

- Los alumnos no tienen un conocimiento exhaustivo del límite finito de una sucesión ya que ningún alumno contesta de manera correcta a las tres preguntas ni justifica de manera adecuada sus respuestas.

\subsection{Tercera etapa. Elaboración del instrumento}

\footnotetext{
${ }^{1} \mathrm{f}(\mathrm{x})$ hace referencia a los valores que toma la sucesión
} 
El Cuestionario tuvo en cuenta, además de todo lo aprendido con el Cuestionario Piloto, las observaciones realizadas por los investigadores presentes en la reunión del grupo de Pensamiento Numérico y Algebraico de la SEIEM (2007), al documento Fenómenos que organizan el límite: diseño de un instrumento (CLAROS, SÁNCHEZ, Y CORIAT, 2007), celebrado en Aravaca (Madrid). El Cuestionario constó de tres preguntas. Los enunciados no coincidieron con los del Cuestionario Piloto pero mantuvieron la estructura. Las instrucciones para la realización se suministraron con el propio cuestionario que, con sus instrucciones, se reproducen en el Anexo 5.

Para la corrección reestructuramos las categorías indicadas en el apartado anterior, como se muestra en el Cuadro 3.

\begin{tabular}{|c|l|}
\hline Categorías & \multicolumn{1}{c|}{ Descripción } \\
\hline C0 & $\begin{array}{l}\text { Calcula correctamente el límite de la sucesión presentada y emplea la justificación } \\
\text { dada por el alumno ficticio }\end{array}$ \\
\hline C1 & $\begin{array}{l}\text { Calcula correctamente el límite de la sucesión presentada y emplea algún fenómeno en } \\
\text { sus justificaciones } \\
\text { Categoría C1.1. Emplea el fenómeno de aproximación simple intuitiva (a.s.i), } \\
\text { representación gráfica y formato ejemplo. } \\
\text { Categoría C1.2. Emplea el fenómeno de aproximación simple intuitiva (a.s.i), } \\
\text { representación tabular y formato ejemplo. } \\
\text { Categoría C1.3. Emplea el fenómeno de aproximación simple intuitiva (a.s.i), } \\
\text { representación verbal y formato ejemplo. } \\
\text { Categoría C1.4 Emplea el fenómeno de retroalimentación o ida-vuelta (i.v.s), } \\
\text { representación verbal y formato ejemplo. }\end{array}$ \\
\hline C2 & $\begin{array}{l}\text { Calcula correctamente el límite de la sucesión presentada, pero no emplea ningún } \\
\text { fenómeno en sus justificaciones } \\
\text { Categoría C2.1 Justifica su respuesta de alguna manera, sin emplear ningún fenómeno. } \\
\text { Categoría C2.2 No justifica su respuesta. }\end{array}$ \\
\hline C3 & $\begin{array}{l}\text { No calcula correctamente el límite. } \\
\text { C 3.1 Usa una idea de infinito potencial. } \\
\text { C 3.2 No usa una idea de infinito potencial. }\end{array}$ \\
\hline C4 & Plantea la necesidad de conocer más valores de la sucesión \\
\hline C5 & No sabe / No contesta \\
\hline
\end{tabular}

Un mayor cuidado en la definición de las categorías era imprescindible porque, a diferencia del estudio de los libros de texto (CLAROS, 2010), no podíamos garantizar que las respuestas a nuestras preguntas fueran matemáticamente correctas. Por esta razón, en el estudio descriptivo nos referimos a las categorías, aunque solamente una de ellas con sus cuatro subcategorías $(\mathrm{C} 1.1, \mathrm{C} 1.2, \mathrm{C} 1.3$ y C1.4) corresponde, efectivamente, a fenómenos.

2 Ejemplo: si el alumno contesta que la sucesión $0,9,0,99,0,999 \ldots$ tiende a $0,99999 \ldots=0, \widehat{\mathbf{9}}$, y no tiende a 1 , ha calculado incorrectamente el límite y maneja una idea de infinito potencial. 


\section{Administración del cuestionario y asignación de categorías}

\subsection{La muestra y su estructura}

El cuestionario se administró en tres Institutos de Enseñanza Secundaria de Madrid: José Hierro (Getafe, Madrid), Celestino Mutis y Dámaso Alonso. El segundo se halla en una zona donde predomina una clase media-baja; los otros dos se hallan en zonas de clase mediaalta. La muestra fue intencional, se eligieron aquellos institutos en los que se tuvo la posibilidad de administrar el Cuestionario. Previamente, entrevistamos a los profesores que participaban en el estudio cediendo su clase; preguntamos, entre otras cosas, si habían explicado ese año el concepto de límite en alguno de los grupos que tenían, y qué grupos tenían asignados. A continuación comentamos que queríamos observar las respuestas de los alumnos ante un cuestionario en el que aparecían cuestiones relativas al límite finito de una sucesión. Después de fijar los días en los que el investigador podía acudir a sus respectivos centros, recibieron una copia del cuestionario.

De los 143 alumnos que participaron, 64 fueron hombres y 79 mujeres. Las edades oscilaron entre 16 y 20 años. Hay que señalar el interés que mostraron los alumnos ante las instrucciones del cuestionario y en la realización de la prueba.

La participación por Centro y grupo se describe en el Cuadro 4.

\begin{tabular}{|c|c|c|c|c|}
\hline Institutos & Fecha de administración & Grupos & & \\
\hline \multirow{4}{*}{ "José Hierro" -JH } & \multirow{4}{*}{ 1/abril/2007 } & $1^{\circ}$ Bachillerato A & 4 & \multirow{4}{*}{7} \\
\hline & & $1^{\circ}$ Bachillerato B & 0 & \\
\hline & & $2^{\circ}$ Bachillerato $\mathrm{B}$ & 2 & \\
\hline & & $2^{\circ}$ Bachillerato $\mathrm{C}$ & 1 & \\
\hline \multirow{2}{*}{ "Celestino Mutis" -CM } & \multirow{2}{*}{ 10/mayo/2007 } & $1^{\circ}$ Bachillerato A & 2 & \multirow[b]{2}{*}{0} \\
\hline & & $1^{\circ}$ Bachillerato B & 8 & \\
\hline \multirow{2}{*}{ "Dámaso Alonso" -DA } & \multirow{2}{*}{ 14/junio/2007 } & $1^{\circ}$ Bachillerato $\mathrm{X}$ & 1 & \multirow[b]{2}{*}{6} \\
\hline & & $1^{\circ}$ Bachillerato Y & 5 & \\
\hline
\end{tabular}

Cuadro 4 - Distribución de sujetos por Centro y grupo y fecha de administración del cuestionario. Los nombres de los Grupos son competencia de cada Instituto.

Éstos eran los grupos en los que los profesores impartían docencia. El cuestionario se administró a un grupo de $2^{\circ}$ de bachillerato de ciencias sociales del IES José Hierro, donde el 
investigador impartía docencia en aquel momento, pero en el cual no se explicó el concepto de límite de una sucesión durante ese curso.

En el proceso de recogida de datos estuvo presente, en todo momento, el investigador, que observó cómo los alumnos realizaban el cuestionario. En cada grupo también estuvo presente el profesor colaborador en el estudio.

Los resultados de las respuestas obtenidas se corrigieron, siguiendo las categorías del Cuadro 3. En Claros (2010) se realizó el recuento de cada categoría en los tres niveles de agregación indicados: Grupo, Centro y Global, incluso se establecieron relaciones entre las respuestas de los alumnos y algunas variables secundarias como la edad o el sexo. Aquí presentamos resultados globales con el propósito de dar una visión general.

\subsection{Ejemplos de asignación de categorías}

El Cuadro 5 incluye ejemplos de respuestas de alumnos y la asignación justificada de categorías hecha por el investigador Se ha optado por transcribir la respuesta junto a la categoría asignada. Las frecuencias se indican más abajo.

\begin{tabular}{|c|c|c|}
\hline $\begin{array}{l}\text { Origen de } \\
\text { la respuesta }\end{array}$ & Transcripción & Asignación justificada \\
\hline $\mathrm{DA}, 1^{\circ} \mathrm{X}$ & $\begin{array}{l}\text { Sí, el límite es 3. La justificación es la que el } \\
\text { alumno ficticio explica en todas las preguntas. }\end{array}$ & $\begin{array}{l}\text { Categoría } \mathrm{C} 0 \text {, ya que emplea la misma } \\
\text { justificación dada por el alumno ficticio. (En } \\
\text { este caso, el empleo corresponde a darla por } \\
\text { buena.) }\end{array}$ \\
\hline $\mathrm{JH}, 2^{\circ} \mathrm{C}$ & $\begin{array}{l}\text { Hace un dibujo. Sí. En la gráfica se va acercando } \\
\text { cada vez más al valor } 3\end{array}$ & $\begin{array}{l}\text { Categoría } \mathrm{C} 1.1 \text {, emplea el fenómeno a.s.i, } \\
\text { usa la representación gráfica y el formato } \\
\text { ejemplo. }\end{array}$ \\
\hline $\mathrm{DA}, 1^{\circ} \mathrm{X}$ & $\begin{array}{l}\text { Es verdadera, ya que cuando } n \text { se va haciendo } \\
\text { más grande, la sucesión se va acercando a } 3 .\end{array}$ & $\begin{array}{l}\text { Categoría C1.3 porque maneja el fenómeno } \\
\text { a.s.i, usa la representación verbal y el } \\
\text { formato ejemplo. }\end{array}$ \\
\hline $\mathrm{JH}, 2^{\circ} \mathrm{B}$ & $\begin{array}{l}\text { Teniendo en cuenta que una sucesión de número } \\
\text { reales } a_{n} \text { tiene por limite el número real a, cuando } \\
\text { para todo número real positivo E existe un número } \\
\text { natural } n^{*} \text {, tal que para todo } n>n * \text { se verifica que } \\
\left|a_{n}-a\right|<\varepsilon \\
\text { Estoy de acuerdo con la afirmación, siempre que } \\
\text { el número al que se reduzca sea menor que el } \\
\text { número } \varepsilon\end{array}$ & $\begin{array}{l}\text { Categoría C1.4 porque surge el fenómeno } \\
\text { i.v.s, usa el sistema de representación } 1 \\
\text { verbal y el formato ejemplo }\end{array}$ \\
\hline $\mathrm{JH}, 1^{\circ} \mathrm{B}$ & $\begin{array}{l}\text { Si porque la } a_{n} \text { tiende a } 1 \text { y nunca va a pasar de } \\
1,5 \text { por lo tanto es un límite }\end{array}$ & $\begin{array}{l}\text { Categoría C } 2.1 \text { porque calcula correctamente } \\
\text { el límite de la sucesión y justifica su } \\
\text { respuesta de alguna manera sin emplear } \\
\text { ninguno de los fenómenos que manejamos } \\
\text { (a.s.i e i.v.s) }\end{array}$ \\
\hline $\mathrm{JH}, 1^{\circ} \mathrm{B}$ & Sí, estoy de acuerdo & $\begin{array}{l}\text { Categoría C2.2 porque el alumno calcula } \\
\text { correctamente el límite de la sucesión } \\
\text { presentada pero no justifica su respuesta. }\end{array}$ \\
\hline
\end{tabular}




\begin{tabular}{|l|l|l|}
\hline $\mathrm{JH}, 2^{\circ} \mathrm{B}$ & $\begin{array}{l}\text { La solución es incorrecta dado que a pesar de sea } \\
\text { cierto que el límite de esta sucesión, y que cuando } \\
\begin{array}{l}\text { crece los valores de la solución se van } \\
\text { acercando a ese número, sin embargo nunca va a a } \\
\text { llegar al número 3 }\end{array}\end{array}$ & $\begin{array}{l}\text { Categoría C3.1 porque no calcula } \\
\text { correctamente el límite y además en su } \\
\text { respuesta aparece el infinito potencial } \\
\text { expresado a través de la frase "nunca va a } \\
\text { llegar al número 3". }\end{array}$ \\
\hline $\mathrm{CM}, 1^{\circ} \mathrm{B}$ & $\begin{array}{l}\text { No estoy de acuerdo; porque no tiene limite pero } \\
\text { el limite no es 3 sino infinito” }\end{array}$ & $\begin{array}{l}\text { Categoría C3.2 porque el alumno no calcula } \\
\text { correctamente el límite de la sucesión } \\
\text { presentada; afirma, erróneamente, que no es } \\
\text { 3. Además no usa el infinito potencial. }\end{array}$ \\
\hline $\mathrm{CM}, 1^{\circ} \mathrm{A}$ & $\begin{array}{l}\text { No, solo con esta gráfica no podríamos afirmar } \\
\text { que el límite de esta sucesión sea 3, habría que } \\
\text { dar valores mucho más altos que 10 para saberlo }\end{array}$ & $\begin{array}{l}\text { Categoría C4, porque el alumno plantea la } \\
\text { necesidad de conocer más valores de la } \\
\text { sucesión. Así interpretamos su texto “... } \\
\text { habría que dar valores mucho más altos...”. }\end{array}$ \\
\hline $\mathrm{CM}, 1^{\circ} \mathrm{B}$ & $\begin{array}{l}\text { a) (Sí, porque) No entiendo su pregunta } \\
\text { b) No entiendo su pregunta }\end{array}$ \\
\hline
\end{tabular}

Cuadro 5 - Ejemplos de asignación de categorías

\section{Resultados}

La Tabla 1 resume los resultados obtenidos.

A cada pregunta del Cuestionario (primera fila) le hemos asociado tres campos: Categoría asignada por el investigador, FRABS (frecuencia absoluta, obtenida como recuento de ocurrencias de la categoría) y \% (muestra la misma información como tanto por ciento, redondeado a la cifra de las unidades). -La abreviatura NHD significa no hay ocurrencia de la categoría; cuando esto ocurre para todas las preguntas, hemos tachado la línea.

Tabla 1 - Resultados agregados

\begin{tabular}{|c|c|c|c|c|c|c|c|c|}
\hline \multirow{2}{*}{ ategoría } & \multicolumn{2}{|c|}{ Pregunta $1^{\mathrm{a}}$} & \multicolumn{2}{|c|}{ Pregunta $2^{a}$} & \multicolumn{2}{|c|}{ Pregunta $3^{a} a$} & \multicolumn{2}{|c|}{ Pregunta $3^{\mathrm{a}} \mathrm{b}$} \\
\hline & RABS & & RABS & & RABS & & RABS & \\
\hline 0 & & & & & & & HD & HD \\
\hline 1.1 & HD & HD & & & HD & HD & & \\
\hline 1.2 & $\mathrm{HD}$ & $\mathrm{HD}$ & $\mathrm{HD}$ & $\mathrm{HD}$ & $\mathrm{HD}$ & $\mathrm{HD}$ & $\mathrm{HD}$ & $\mathrm{HD}$ \\
\hline 1.3 & 0 & 9 & 0 & 9 & 2 & 6 & 7 & 9 \\
\hline 1.4 & & & & & & & & \\
\hline 2.1 & 3 & 3 & 4 & 4 & 4 & 4 & 1 & \\
\hline 2.2 & 1 & & 3 & & 5 & 0 & 9 & 3 \\
\hline 3.1 & & & & & & & & \\
\hline 3.2 & 5 & 0 & 0 & & 2 & & 9 & 0 \\
\hline 4 & & & & & HD & HD & & \\
\hline & & & & & & & & \\
\hline
\end{tabular}




\begin{tabular}{c|c|c|c|c|c|c|c|c}
\hline 5 & & & & & 5 & 0 & 1 & 9 \\
\hline \multirow{2}{*}{ OT } & 43 & 00 & 43 & 00 & 43 & 00 & 43 & 00 \\
\hline
\end{tabular}

Comentamos respuestas más frecuentes, respuestas menos frecuentes y otros resultados.

\section{Respuestas más frecuentes}

En la pregunta ${ }^{\circ}$ 1, la categoría C1.3 es la más frecuente (70), seguida de la categoría C2.1, con una frecuencia de 33. En la pregunta $n^{\circ} 2$ vuelve a ser la categoría C1.3 la más frecuente (70), seguida de la categoría C2.1 con una frecuencia de 34 . En la pregunta $\mathrm{n}^{\mathrm{o}} 3$ (a), la categoría $\mathrm{C} 1.3$, es la categoría más frecuente (52), seguida de la categoría C2.1, con una frecuencia de 34. En pregunta $n^{\circ} 3$ (b), la categoría C5 la que ocupa la primera posición, con una frecuencia de 41, seguida por la categoría C3.2 con una frecuencia de 29.

\section{Respuestas menos frecuentes (frecuencia 1)}

En la pregunta $\mathrm{n}^{\mathrm{o}} 1$, son las categorías C1.4 y C5 las que tienen menor frecuencia. En la pregunta $\mathrm{n}^{\mathrm{o}} 2$, las categorías C1.1, C1.4, C3.1 y C4. En la pregunta $\mathrm{n}^{\mathrm{o}} 3$ (a), la respuesta menos frecuente es aquella que usa la categoría C1.4. En la pregunta $n^{\circ} 3$ (b), son las categorías C1.1, C3.1 y C4 las menos usuales en las respuesta de los alumnos.

\section{Otros resultados}

- No existen diferencias notables en las respuestas de mayor frecuencia en lo relativo a las preguntas $1^{\mathrm{a}}$ y $2^{\mathrm{a}}$ y $3^{\mathrm{a}}(\mathrm{a})$ a pesar de usar diferentes sistemas de representación aunque en los tres casos se emplee el fenómeno de aproximación simple intuitiva en su enunciado. La respuesta más frecuente relativa a la pregunta $3^{\mathrm{a}}(\mathrm{b})$, en cambio, deja al descubierto el menor conocimiento relativo de la definición formal de límite finito ya que un $29 \%$ de los alumnos no sabe/no contesta a dicha cuestión.

- El fenómeno de aproximación simple intuitiva parece ayudar a los alumnos a establecer correctamente el límite de la sucesión, a juzgar por los altos porcentajes de acierto (82, en la pregunta $1^{\mathrm{a}}, 85$, en la pregunta $2^{\mathrm{a}}$ y 74 , en la pregunta $3^{\mathrm{a}}$ (a)). Por otro lado cuando se ven abocados a usar el fenómeno de retroalimentación, el porcentaje de aciertos se reduce: $49 \%$ en la pregunta $3^{\mathrm{a}}(\mathrm{b})$.

- La categoría C5 va aumentando su frecuencia a medida que avanzamos en la prueba; pasa, sucesivamente, por los valores 1, 9, 15 y 41, para las preguntas en el orden de presentación. Sobre este resultado cabe enunciar dos consideraciones: $\left(1^{\text {a }}\right)$ La comprensión del enunciado no parece implicar una respuesta correcta; así, en la pregunta $1^{\mathrm{a}}$, con una sola ocurrencia de la categoría C5, hay un porcentaje menor de respuestas correctas que en la 
pregunta $2^{\mathrm{a}}$, a pesar de que, en ésta, la misma categoría se dio 9 veces. (2a) La representación gráfica podría ser la que más sirvió a los alumnos para comprender el enunciado.

- E 1 uso que hacen los alumnos, del fenómeno de ida-vuelta en sucesiones o i.v.s (categoría C1.4) en sus respuestas es casi inexistente. Su frecuencia es de 1 (pregunta $1^{\mathrm{a}}$, pregunta $2^{\mathrm{a}}$, pregunta $3^{\mathrm{a}}(\mathrm{a})$ ) y de 3 (pregunta $3^{\mathrm{a}}(\mathrm{b})$ ); esto corresponde a 6 respuestas de un total de 572. En todos estos casos, usaron el sistema de representación verbal y el formato ejemplo.

- El uso del fenómeno de aproximación simple intuitiva o a.s.i (categorías C1.1, C1.2 y C1.3) supera al fenómeno i.v.s (C1.4). Si, por argumentos de equidad, estimamos la razón de uso esperada en 3:1, las que efectivamente observamos son: 70:1 (pregunta $1^{\mathrm{a}}$ ), 71:1 $\left(\right.$ pregunta $2^{\mathrm{a}}$ ), 52:1 (pregunta $\left.3^{\mathrm{a}}(\mathrm{a})\right)$ y $28: 3\left(\right.$ pregunta $\left.3^{\mathrm{a}}(\mathrm{b})\right)$.

- La categoría C1.3 es la más frecuente (219 de 572). Deducimos que el fenómeno de aproximación simple intuitiva en el sistema de representación verbal y en el formato ejemplo es el más usado por los alumnos en sus respuestas.

\section{Comparación de resultados entre el cuestionario y los libros de texto}

En Claros (2010) y Claros, Sánchez y Coriat (2013, pendiente de publicación) estudiamos y observamos los fenómenos de aproximación simple intuitiva (a.s.i) y retroalimentación o ida-vuelta en sucesiones (i.v.s) en 30 libros de texto de diferentes periodos educativos. Los resultados obtenidos en dichos trabajos se sintetizan en las tablas 2 y 3.

Tabla 2 - Aproximación simple intuitiva y periodo educativo

\begin{tabular}{l|c}
\hline \multicolumn{1}{c|}{ Fenómeno } & $\begin{array}{c}\text { Periodo 1995- } \\
2005\end{array}$ \\
\hline $\begin{array}{l}\text { Aproximación simple intuitiva, en el sistema de representación verbal y en el } \\
\text { formato ejemplo }\end{array}$ & 9 \\
\hline $\begin{array}{l}\text { Aproximación simple intuitiva, en el sistema de representación verbal y en el } \\
\text { formato definición }\end{array}$ & 5 \\
\hline $\begin{array}{l}\text { Aproximación simple intuitiva, en el sistema de representación tabular y en el } \\
\text { formato ejemplo }\end{array}$ & 11 \\
\hline $\begin{array}{l}\text { Aproximación simple intuitiva, en el sistema de representación tabular y en el } \\
\text { formato definición }\end{array}$ & 0 \\
\hline $\begin{array}{l}\text { Aproximación simple intuitiva, en el sistema de representación gráfico y en el } \\
\text { formato ejemplo }\end{array}$ & 13 \\
\hline $\begin{array}{l}\text { Aproximación simple intuitiva, en el sistema de representación gráfico y en el } \\
\text { formato definición }\end{array}$ & 0 \\
\hline $\begin{array}{l}\text { Aproximación simple intuitiva, en el sistema de representación simbólico y en el } \\
\text { formato ejemplo }\end{array}$ & 0 \\
\hline $\begin{array}{l}\text { Aproximación simple intuitiva, en el sistema de representación simbólico y en el } \\
\text { formato definición }\end{array}$ & 0 \\
\hline
\end{tabular}


Tabla 3 - Retroalimentación o ida-vuelta en sucesiones y periodo educativo

\begin{tabular}{l|c}
\hline \multicolumn{1}{c|}{ Fenómeno } & $\begin{array}{c}\text { Periodo 1995- } \\
2005\end{array}$ \\
\hline $\begin{array}{l}\text { Ida-vuelta en sucesiones en el sistema de representación verbal y en el formato } \\
\text { ejemplo }\end{array}$ & 2 \\
\hline $\begin{array}{l}\text { Ida-vuelta en sucesiones en el sistema de representación verbal y en el formato } \\
\text { definición }\end{array}$ & 4 \\
\hline $\begin{array}{l}\text { Ida-vuelta en sucesiones en el sistema de representación tabular y en el formato } \\
\text { ejemplo }\end{array}$ & 0 \\
\hline $\begin{array}{l}\text { Ida-vuelta en sucesiones en el sistema de representación tabular y en el formato } \\
\text { definición }\end{array}$ & 2 \\
\hline $\begin{array}{l}\text { Ida-vuelta en sucesiones en el sistema de representación gráfico y en el formato } \\
\text { ejemplo }\end{array}$ & 0 \\
\hline $\begin{array}{l}\text { Ida-vuelta en sucesiones en el sistema de representación gráfico y en el formato } \\
\text { definición }\end{array}$ & 2 \\
\hline $\begin{array}{l}\text { Ida-vuelta en sucesiones en el sistema de representación simbólico y en el } \\
\text { formato ejemplo }\end{array}$ & 0 \\
\hline $\begin{array}{l}\text { Ida-vuelta en sucesiones en el sistema de representación simbólico y en el } \\
\text { formato definición }\end{array}$ & 10 \\
\hline Recuento total & 2 \\
\hline
\end{tabular}

Comparamos los resultados del cuestionario y los resultados de los libros de texto del periodo 1995-2005 (por ser el periodo en el que se encontraban inmersos los alumnos).

El fenómeno de mayor frecuencia es, en los libros de texto de ese periodo, la aproximación simple intuitiva en el sistema de representación gráfico y en el formato ejemplo, con 13 ocurrencias, mientras que en las respuestas de los alumnos, lo es el fenómeno de aproximación simple intuitiva verbal ejemplo con 26 ocurrencias. No sabemos explicar este cambio de la representación gráfica a la representación verbal ni disponemos de información que lo explique. En las producciones de los alumnos, la representación gráfica se observó solamente en 2 de las 572 respuestas recogidas.

La categoría C.1.3 (fenómeno de aproximación simple intuitiva en el sistema de representación verbal y en el formato ejemplo) es la más usada por los alumnos en sus respuestas a las preguntas $1^{\mathrm{a}}, 2^{\mathrm{a}}$ y $3^{\mathrm{a}}$ (a); las correspondientes ocurrencias suman casi la mitad del total (219 de 572). Esta diferencia en las ocurrencias de los fenómenos preferidos (en los libros de texto, el fenómeno de aproximación simple intuitiva en el sistema de representación gráfico y en el formato ejemplo, y en las producciones de los alumnos el fenómeno de aproximación simple intuitiva en el sistema de representación verbal y en el formato ejemplo) conduce a conjeturar que, siendo de importancia, la influencia de los libros de texto en las producciones de los alumnos no es determinante.

A pesar de que el fenómeno de aproximación simple intuitiva en el sistema de representación tabular y en formato ejemplo, se observa con bastante frecuencia en los libros 
de texto (es el segundo con mayor frecuencia, después del fenómeno de aproximación simple intuitiva en el sistema de representación gráfico y en el formato ejemplo), no es usado por los alumnos para justificar sus respuestas en ninguna ocasión. Sin embargo el uso de este fenómeno en el enunciado de la pregunta ayuda a responder de manera adecuada a la cuestión presentada. De hecho la cuestión número dos, en la que se empleó dicho fenómeno, fue la que obtuvo el mayor porcentaje de respuestas correcta $(85 \%)$.

El descenso del fenómeno de retroalimentación o ida-vuelta en sucesiones se ha manifestado tanto en los libros de texto como en las respuestas de los alumnos; si en el estudio de libros de textos la relación entre fenómenos de retroalimentación y aproximación simple intuitiva es 10:38, en las respuestas del cuestionario dicha relación es 6:221.

El sistema de representación verbal es el más usado por los alumnos para justificar sus respuestas, ya sea cuando eligen el fenómeno de aproximación simple intuitiva ya sea cuando eligen el fenómeno de retroalimentación o ida-vuelta en sucesiones: 198 respuestas de 572 posibles. En los libros de texto, dicho sistema de representación es también el más empleado tanto en el fenómeno de aproximación simple intuitiva como en el fenómeno de retroalimentación o ida-vuelta en sucesiones

\section{Conclusiones y perspectivas}

Los fenómenos de aproximación simple intuitiva y retroalimentación o i.v.s son usados en las respuestas de los alumnos para justificar el hecho de que una determinada sucesión tenga límite.

El uso del fenómeno de aproximación simple intuitiva en los sistemas de representación verbal, gráfico y tabular favorece la comprensión de las preguntas del Cuestionario y ayuda a contestar de manera correcta a ellas.

El uso del fenómeno de retroalimentación o ida-vuelta en sucesiones en este caso en el sistema de representación verbal, no ayuda a responder de manera correcta a la pregunta que se presenta en el Cuestionario.

Los alumnos usan con mayor frecuencia el fenómeno de aproximación simple intuitiva (a.s.i) en sus respuestas, para justificar que la sucesión presentada tiene límite.

El sistema de representación más usado por los alumnos para justificar sus respuestas es el sistema de representación verbal. 
La secuencia lógica definición de límite finito de una sucesión-libros de texto-alumnos parece cumplirse de manera adecuada. Es decir, a lo largo más de 13 años de investigación observamos cómo el límite finito de una sucesión organizaba dos fenómenos que denominamos fenómeno de aproximación simple intuitiva o a.s.i y fenómeno de retroalimentación o i.v.s (CLAROS, 2010; CLAROS; SÁNCHEZ; CORIAT, 2013). Estos fenómenos estaban presentes en las definiciones y posterior desarrollo de las mismas que los libros hacían del límite finito de una sucesión (CLAROS; SÁNCHEZ; CORIAT, 2013, pendiente de publicación). Después de este estudio confirmamos el uso de estos fenómenos por los alumnos cuando tienen que justificar que una determinada sucesión tiene límite.

Cuando abordamos la realización del Cuestionario y su análisis nos basamos en la suposición de que al estar los fenómenos de aproximación simple intuitiva y el fenómeno de ida-vuelta en sucesiones en los libros de texto y puesto que los profesores de secundaria usan muy a menudo el libro de texto, dichos fenómenos debían, posiblemente, estar presentes en las respuestas y justificaciones de los alumnos ante cuestiones relativas al límite finito de una sucesión.

No hemos abordado en la secuencia anterior la figura del profesor. Queda por estudiar el hecho de que el profesor use estos fenómenos para explicar el límite finito de una sucesión.

El estudio con profesores sobre el uso de los fenómenos de aproximación simple intuitiva y retroalimentación o ida-vuelta en sucesiones debe ayudar a establecer perfiles fenomenológicos de los mismos, los cuales fueron descritos por SÁNCHEZ (2012) en el caso del límite finito de una función en un punto. Este estudio queda pendiente y completará la secuencia mencionada.

\section{Referencias}

BLÁZQUEZ, S.; ORTEGA, T. El concepto de límite en la educación secundaria. In: CANTORAL, R. (Ed.). En el futuro del cálculo infinitesimal. México: Grupo Editorial Iberoamérica. 2000. p.331354.

CLAROS, F. J.; SÁNCHEZ, M. T.; CORIAT, M. Fenómenos que organizan el límite. In: BOLEA, P; CAMACHO, M; FLORES, P; GÓMEZ, B; MURILLO, J; GONZÁLEZ, M.T; SIMPOSIO DE LA SOCIEDAD ESPAÑOLA DE INVESTIGACIÓN EN EDUCACIÓN MATEMÁTICA SEIEM. 10., 2006, Huesca. Actas... Zaragoza: Universidad de Zaragoza, 2006. p. 157-172.

CLAROS, F. J.; SÁNCHEZ. M. T.; CORIAT, M. Fenómenos que organizan el límite: diseño de un instrumento. Indivisa, boletín de estudios de investigación. MADRID, n.9, p.49-68, octubre. 2007.

CLAROS, F. J. Límite finito de una sucesión: fenómenos que organiza. 2010. 441f. Tesis (Doctorado en Educación Matemática) - Facultad de Ciencias de la Educación, Universidad de Granada. Granada, 2010. 
CLAROS, J. SÁNCHEZ, M. T.; CORIAT, M. Sucesión convergente y sucesión de Cauchy: hacia una secuencia didáctica basada en la fenomenología. Enseñanza de las Ciencias, Barcelona, v. 31, n. 2, p. 113-131, mayo. 2013.

CORNU, B. Limits. In: TALL, D. (ed.). Advanced Mathematical Thinking. Dordrecht: Kluwer, 1991. p. 153-166.

DREYFUS, T. Advanced mathematical thinking. In: NESHER, P.; KILPATRICK, J. (Ed.).

Mathematics and cognition. Cambridge, U. K.: Cambridge University Press. 1990. p. 113-133.

EDWARDS, B. S.; DUBINSKY, E.; MCDONALD, M. A. Advanced Mathematical Thinking. Mathematical Thinking and Learning. Hillsdale, N. J.: Lawrence Erlbaum Associates, 2005. p. 1525 .

FREUDENTHAL, H. Didactical Phenomenology of Mathematical Structures. Dordrecht: Reidel Publishing Company, 1983

GUCLER. B. Development of discourse on limits: connecting history and classroom practice through a communicational approach to learning. 2010. 259 f. Tesis (Doctorado en Filosofía, Educación Matemática) - Departamento de STEM, Universidad de Michigan, Michigan, 2010.

JANVIER, C. Problems of Representation in the Teaching and Learning of Mathematics. Hillsdale, N. J.: Lawrence Erlbaum Associates, 1987.

SÁNCHEZ, M. T. Límite finito de una función en un punto: fenómenos que organiza. 2012.517 f. Tesis (Doctorado en Educación Matemática) - Departamento de Didáctica de la Matemática, Facultad de Ciencias de la Educación, Universidad de Granada, Granada, 2012.

SPIVAK, M. Calculus. Barcelona: Reverté. 1991.

TALL, D. Advanced mathematical thinking. Dordrecht: Kluwer. 1991.

Submetido em Fevereiro de 2014. Aprovado em Julho de 2014. 


\section{Anexo 1. Cuestionario Inicial}

\section{Pregunta 1.}

Se representa gráficamente la sucesión $b_{n}=1+1 / n$. El profesor pregunta a la clase: ¿Tiene límite la sucesión? Un alumno responde: "El límite es 1 porque a medida que $\mathrm{n}$ crece los valores de la sucesión se van acercando cada vez más a 1". ¿Estás de acuerdo con él? Justifica tu respuesta.

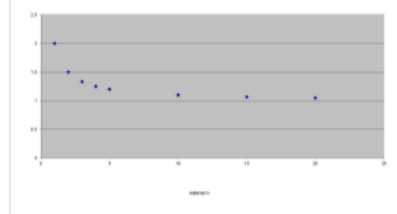

Pregunta 2.

Se representa gráficamente la sucesión $b_{n}=3-1 / n$. El profesor pregunta a la clase: ¿Tiene límite la sucesión? Un alumno responde: "El límite es 3 porque a medida que n crece los valores de la sucesión se van acercando cada vez más a 3". ¿Estás de acuerdo con él? Justifica tu respuesta.

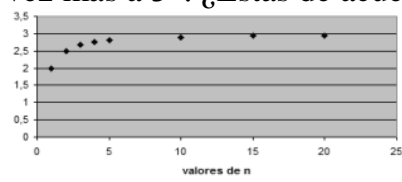

Pregunta 3

Las ganancias de una empresa que últimamente marcha muy mal económicamente vienen representadas por la siguiente gráfica:

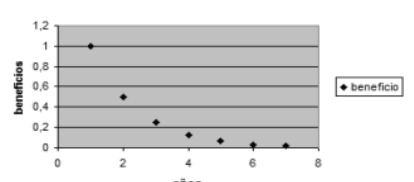

El profesor pregunta: ¿Llegará a ser el beneficio nulo? El alumno responde: "El límite del beneficio es 0, porque a medida que aumente el tiempo, el beneficio disminuye”. ¿Estás de acuerdo con él? Justifica tu respuesta.

Pregunta 4.

La velocidad de una nave espacial, va aumentando siguiendo la siguiente gráfica:

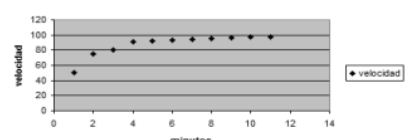

El profesor pregunta ¿Llegará a alcanzar la nave la velocidad de $100 \mathrm{~km} /$ minuto? Un alumno responde: 'No, está cada vez más cerca de $100 \mathrm{~km} /$ minuto, pero nunca llega a alcanzarla". ¿Estás de acuerdo con él? Justifica tu respuesta.

\section{Pregunta 5.}

Dada la siguiente sucesión $\mathrm{a}_{\mathrm{n}}$, expresada a través de una tabla:

\begin{tabular}{|l|l|}
\hline $\mathrm{n}$ & $\mathrm{a}_{\mathrm{n}}$ \\
\hline 1 & $1 / 5$ \\
\hline 2 & $1 / 25$ \\
\hline 3 & $1 / 125$ \\
\hline$\ldots$. & $\ldots$. \\
\hline
\end{tabular}

¿A qué valor se aproximan los valores de la sucesión a medida que continuamos dando valores a n? ¿Es este valor el límite de la sucesión? Justifica tu respuesta.

Pregunta 6.

Dada la siguiente sucesión:

\begin{tabular}{|l|l|}
\hline $\mathrm{n}$ & $\mathrm{A}_{\mathrm{n}}$ \\
\hline 1 & 1.9 \\
\hline 2 & 1.99 \\
\hline 3 & 1.999 \\
\hline
\end{tabular}




\section{\begin{tabular}{|l|l|}
\hline$\cdots$ & $\cdots . .$. \\
\hline
\end{tabular}}

¿A qué valor se aproximan los valores de la sucesión a medida que continuamos dando valores a n? ¿Es este valor el límite de la sucesión? Justifica tu respuesta.

Pregunta 7

Tenemos un cuadrado de lado $1 \mathrm{~m}$ y dividimos el cuadrado en cuatro partes. Cada cuadrado resultante lo divido en 4 partes y así sucesivamente. La siguiente tabla recoge las medidas de cada cuadrado, resultante de la división de un cuadrado de lado $1 \mathrm{~m}$ :

\begin{tabular}{|l|l|}
\hline $\mathrm{n}$ & $\mathrm{a}_{\mathrm{n}}$ \\
\hline 1 & 1 \\
\hline 2 & $1 / 4$ \\
\hline 3 & $1 / 16$ \\
\hline$\ldots$. & $\ldots$. \\
\hline
\end{tabular}

El profesor pregunta a un alumno: ¿Tiene límite la sucesión considerada? El alumno responde "El límite de la sucesión resultante de cortar el cuadrado es cero”. ¿Estás de acuerdo con el? Justifica tu respuesta.

Pregunta 8

Una persona debe recoger andando los $10 \mathrm{~km}$, que separan dos ciudades. Cada día va a recorrer la mitad del camino que le falta para llegar al final. La siguiente tabla recoge el espacio recorrido hasta el día $\mathrm{n}$.

\begin{tabular}{|l|l|}
\hline $\mathrm{n}$ & $\mathrm{a}_{\mathrm{n}}$ \\
\hline 1 & 5 \\
\hline 2 & $7^{\prime} 5$ \\
\hline 3 & $8^{\prime} 75$ \\
\hline 4 & $9^{\prime} 375$ \\
\hline$\ldots$. & $\ldots$. \\
\hline
\end{tabular}

Miguel responde "Llegará algún día en que recorra la distancia que separa las dos ciudades” ¿Estás de acuerdo con él? Justifica tu respuesta.

Pregunta 9

La sucesión $a_{n}=\frac{1}{10^{n}}$ cumple: su primer término vale $1 / 10$, su segundo término vale $1 / 100$, su tercer término vale 1/1000 y así sucesivamente. Miguel dice: "Esta sucesión no tiene límite, porque nunca voy a parar" ¿Estás de acuerdo con él? Justifica tu respuesta

Pregunta 10

La sucesión siguiente cumple: su primer término vale 3'9, su segundo término vale 3'99, su tercer término vale 3’999, su cuarto término 3’9999 y así sucesivamente. Miguel dice: "Esta sucesión tiene límite 4, porque a medida que avanza n su valor va acercándose a 4”. ¿Estás de acuerdo con él? Justifica tu respuesta

Pregunta 11

La cantidad de agua almacenada en un deposito disminuye siguiendo la siguiente sucesión:

$$
1, \frac{1}{2}, \frac{1}{3}, \frac{1}{4}, \ldots . .
$$

El profesor pregunta: ¿Llegará a vaciarse completamente el depósito? Un alumno responde: “El límite de la capacidad del depósito es 0 , porque a medida que pasa el tiempo, la capacidad se va acercando a 0 ” ¿Estás de acuerdo con él? Justifica tu respuesta.

\section{Pregunta 12}

El enriquecimiento de una persona sigue la siguiente sucesión, medida en millones de euros: 8`9, 8’99, 8’999, 8’9999, ...

¿Llegará a alcanzar alguna vez la cantidad de 9 millones de euros? ¿Es este valor el límite de la sucesión? Un alumno responde: "El límite de su enriquecimiento es 9, porque a medida que n crece su valor va aumentando". ¿Estás de acuerdo con él? Justifica tu respuesta 


\section{Anexo 2. Respuestas de expertos}

Los profesores serán denotados por las letras A, B, C y D para garantizar su anonimato.

\section{Profesor A}

Este profesor no realiza ninguna crítica a las primeras dos cuestiones presentadas. Emplea el signo “+” en cada una de ellas para afirmar que las considera adecuadas.

A la tercera pregunta, realiza una crítica que citamos a continuación de manera textual: "La marcha de una empresa puede cambiar: no responde a una función matemática. No tiene sentido hablar de límite en un número finito ( y pequeño) de "años"."

A la cuarta pregunta realiza la siguiente crítica: “Es una pregunta de contexto "real”?

Las preguntas 5 y 6 son consideradas adecuadas por él. Emplea un signo “+” para confirmar que está de acuerdo con ellas.

En la pregunta $n^{\circ} 7$ considera que la palabra "medidas" que aparece en el enunciado del problema podría ser sustituida por la palabra "área".

En la pregunta $\mathrm{n}^{\circ} 8$ realiza la siguiente anotación: "el número de días ¿puede ser infinito? ¿se puede "recorrer andando" 0,9765625 m (el décimo día)?"

La pregunta $\mathrm{n}^{\circ} 9$ es considerada adecuada por él. Emplea un signo "+" para confirmar que está de acuerdo con ellas.

En la pregunta $\mathrm{n}^{\mathrm{o}} 10$ realiza la siguiente anotación: "igual que la pregunta 6"

En la pregunta $\mathrm{n}^{\circ} 11$ realiza la siguiente anotación: “¿la sucesión indica tiempos iguales?

En la pregunta $\mathrm{n}^{\circ} 12$ realiza la siguiente anotación: "millones de euros $\Rightarrow$ solo tiene sentido 8 decimales. A partir de aquí no tiene sentido el problema. Alcanzará 899999999 céntimos de $€$."

\section{Profesora B}

La profesora B no hace una objeción específica a las preguntas 1 y 2 . Solo comenta en la misma hoja del cuestionario donde aparecen las preguntas 1 y 2, la siguiente observación "se podría añadir un ej. de suc. que no converja ej $a_{n}=(-1)^{n}$ “

A la pregunta 3 hace las siguientes observaciones: “¿Responde a la pregunta? ¿Podría llegar a tener pérdidas?"

A la pregunta 4 realiza la siguiente observación: "Parece que este punto no mantiene la misma tendencia" Esta observación se refiere al gráfico presentado. En él, el valor (3, f(3)), parece no seguir el mismo orden de crecimiento que los demás puntos. En realidad se trata solamente de un efecto óptico, porque la gráfica presentada crece de la misma manera en todo su dominio. También se realiza la siguiente observación “suponiendo una aceleración constante”. Esta observación se realiza a la pregunta que presentamos ¿Llegará a alcanzar la nave la velocidad de $100 \mathrm{~km} /$ minuto?

No se hace ninguna observación a las preguntas 8,9 y 10 .

A la pregunta número 11, realiza la siguiente observación: “¿Responde a la pregunta inicial?”, refiriéndose a la respuesta que presentamos como dada por un alumno.

A la pregunta número 12 realiza la siguiente observación: “sucesión finita: unidades monetarias”, afirmando las dificultades de manejar unidades monetarias con tanto decimales.

\section{Profesora C}

La profesora $\mathrm{C}$ no hace ninguna objeción específica a las preguntas 1, 2, 3, 4, 5 y 6.

A la pregunta número 7, realiza la siguiente observación "del lado" o "del área", refiriéndose a la frase que aparece en la pregunta "la siguiente tabla recoge las medidas de cada cuadrado, resultante de la división de un cuadrado de lado $1 \mathrm{~m}$ ". La profesora no entiende si las medidas se refieren a áreas o a medidas de los lados.

A la pregunta número 8, la profesora realiza la siguiente observación: “ $a_{n}$ espacio recorrido hasta el día $n$ ”, refiriéndose a la imposibilidad de hallar una expresión algebraica que dé el término general. También anota que la palabra "recoger" que aparece en la primera frase de la pregunta deber ser sustituida por "recorrer".

A las preguntas número 9 y 10 no hace ninguna observación.

A la pregunta número 11 realiza la siguiente observación: la palabra "capacidad" que aparece en el enunciado de la pregunta debe ser sustituida por la palabra "almacenada".

A la pregunta número 12 no hace ninguna observación.

\section{Profesor D}

El profesor D realiza los siguientes comentarios que quedan recogidos de manera literal.

"Tarea 1. Quitaría el fondo gris y las líneas de cuadrícula horizontales porque pueden "forzar" la idea de que se alcanza el valor $1(;$ ?).

Tarea 2. La considero exactamente igual que la tarea 1 para los propósitos del estudio. 
Tarea 3. Tiene un aspecto que la diferencia de las tareas 1 y 2 y no es precisamente el poseer un "enunciado de la vida real". Me refiero a que en ella NO aparece el término general de la sucesión en términos simbólicos. Pienso que este hecho podría generar diferencias importantes. En las tareas 1 y 2 el alumno puede apoyarse en la ley que regula la sucesión para afirmar que nunca alcanza el límite. Esta opción no se tiene en la tarea 3.

Tarea 4. La respuesta dada por el alumno ficticio a la tarea 1 ("el límite es 0"), tarea 2 ("el límite es 3") y tarea 3 ("el límite es 1"), no es de la misma naturaleza que la respuesta dada en la tarea 4 ("el límite nunca se alcanza”). ¿Por qué este cambio repentino en la secuencia de tareas?

Tarea 5. Si en las tareas anteriores le presentas a los alumnos hasta 11 valores de la sucesión (véase tarea 4, por ejemplo), ¿por qué ahora tan sólo presentas 3? Deberías unificar este aspecto en todas las tareas. Para la tarea 5, incluiría más valores para que se viera mejor la evolución experimentada por términos de la sucesión. Por otra parte, las cuatro primeras tareas son equivalentes (salvo por lo comentado al hablar de la tarea 4) porque el formato y el contenido de las preguntas es el mismo. ¿Por qué ahora se cambian las cuestiones de formato?; ¿por qué no sigue habiendo un "profesor" y un "alumno" ficticios?; ¿por qué ahora no se les "adelanta” a los chicos cuál es el límite, como en los otros casos?

Tarea 6. ¿Por qué decimal y antes fracción? T5 y T6 son equivalentes y no creo que T6 aporte nada nuevo a T5. Tarea 7. Se vuelve de nuevo al formato de pregunta adoptado en T1, T2, T3 y T4.

Tarea 8. No es verdad que “la siguiente tabla recoge el espacio recorrido hasta el día n”. ¿Dónde está la relación $\left(n, a_{n}\right)$ ?, es decir, no proporcionas el término general de la sucesión.

Tarea 9. ¿Realmente el enunciado está expresado en un sistema de representación verbal? Te lo digo porque presentas el término general de la sucesión (la ley) simbólicamente.

Tarea 10. Si observas, aquí ya no presentas formalmente el término general de la sucesión, a diferencia que en la tarea 9.

Tarea 11 y 12. No me queda claro que tan verbal es el sistema de representación empleado. A mí me parece más próximo al tabular".

\section{Anexo 3. Cuestionario Piloto}

Cuestión $\mathrm{n}^{\circ}$ 1: Se representa gráficamente una sucesión El profesor pregunta a la clase: ¿Tiene límite la sucesión? Un alumno responde: "El límite es 1 porque a medida que $n$ crece los valores de la sucesión se van acercando cada vez más a 1” ¿Estás de acuerdo con él? Justifica tu respuesta.

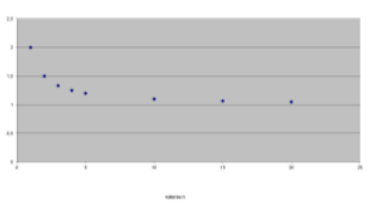

Cuestión $\mathrm{n}^{\mathrm{o}}$ 2: Dada la siguiente sucesión:

\begin{tabular}{|l|l|}
\hline $\mathbf{n}$ & \multicolumn{1}{|c|}{$\mathbf{a}_{\mathbf{n}}$} \\
\hline 1 & 1.9 \\
\hline 2 & 1.99 \\
\hline 3 & 1.999 \\
\hline 4 & 1.9999 \\
\hline 5 & 1.99999 \\
\hline 6 & 1.999999 \\
\hline 7 & 1.9999999 \\
\hline 8 & 1.99999999 \\
\hline$\ldots$ & $\ldots$ \\
\hline
\end{tabular}

El profesor pregunta a la clase: ¿Tiene límite la sucesión? Un alumno responde: "El límite es 2, porque a medida que n crece los valores de la sucesión se van acercando cada vez más a 2" ¿Estás de acuerdo con él? Justifica tu respuesta.

Cuestión $n^{\circ}$ 3: La sucesión siguiente cumple: su primer término vale 3.9, su segundo término vale 3.99, su tercer término vale 3.999, su cuarto término 3.9999, su quinto término 3.99999, su sexto término 3.999999, su séptimo término 3.9999999, su octavo término 3.99999999 y así, sucesivamente.

Un alumno afirma: "Esta sucesión tiene límite 4, porque a medida que avanza n su valor va acercándose cada vez más a 4" ¿Estás de acuerdo con él? Justifica tu respuesta

Otro alumno afirma: "Esta sucesión tiene límite 4, porque para cualquier entorno de 4 que tomemos, se cumple que existe un término de la sucesión a partir del cual todos los términos de la sucesión quedan dentro de ese entorno” ¿Estás de acuerdo con él? Justifica tu respuesta. 


\section{Anexo 4: Categorías de análisis y estudio de las respuestas al Cuestionario Piloto}

Tabla A4-1. Prueba piloto: frecuencias de las categorías
\begin{tabular}{|c|c|}
\hline Categorías & Frecuencias \\
\hline C1 & 13 \\
\hline C 2 & 4 \\
\hline C 3 & 1 \\
\hline C 4 & 3 \\
\hline C5 & 2 \\
\hline C6 & 11 \\
\hline
\end{tabular}

-Observamos que C1 es la categoría más frecuente (13 ocurrencias) y C3 es la menos frecuente (1).

-La categoría $\mathrm{C} 1$, en la que el alumno emplea la respuesta dada por el alumno ficticio, es la más frecuentemente asociada a las respuestas dadas por los alumnos, principalmente, en las preguntas $1^{\mathrm{a}}$ y $2^{\mathrm{a}}$.

- La categoría C3, en la que el alumno identifica el enunciado de la pregunta con la definición de límite solamente se presenta en una de las respuestas de los alumnos.

-Un alumno, de los 8, contestó correctamente a las tres preguntas (con la excepción del apartado b de la pregunta

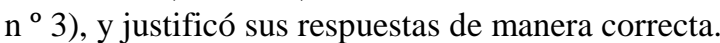

-Solamente hay dos alumnos que se plantea la necesidad de conocer más valores de la sucesión para asegurar con certeza cuál es su límite (categoría C5)

- Hay muchos alumnos que no responden a alguna pregunta del cuestionario, principalmente la pregunta tercera apartado b. (categoría C6)

Las conclusiones que se obtuvieron de las respuestas obtenidas fueron las siguientes:

-Muchos alumnos emplean la respuesta dada por el alumno ficticio como justificación de sus respuestas. . Esto condujo a adoptar un cambio en la redacción de las condiciones de la prueba, que implicó incluir la siguiente recomendación: "preferimos que, en las respuestas, uses tus propias frases para explicar si la solución tiene limite o no.

-Muy pocos alumnos contestan de manera adecuada, es decir responden bien y justifican su respuesta, a las cuestiones presentadas. Este hecho lleva a suponer que serán pocos los alumnos que tengan un conocimiento exhaustivo y sin lagunas del límite finito de una sucesión.

- Solamente dos alumnos plantearon la necesidad de conocer más valores de la sucesión. Con la idea de no sobrecargar el enunciado de las cuestiones, se decidió

que la información se daría solamente a aquellos alumnos que planteasen la necesidad de conocer más valores de la sucesión y no a todos en general.

-Los alumnos no manejan el concepto de entorno. Esto hace que no entiendan el enunciado dado en la pregunta $\mathrm{n}$ - 3, apartado b y que contesten todos "no sabe / no contesta". Esto obliga bien a evitar o bien a explicar la palabra ‘entorno' cuando empleemos el fenómeno de retroalimentación.

Las conclusiones y resultados obtenidos permiten valorar el cumplimiento o no de los objetivos propuestos con el Cuestionario Piloto. El cumplimiento o no de los objetivos propuestas arroja luz sobre la elaboración del nuevo cuestionario:

-No hemos comprobado si se ha cumplido el objetivo general $1^{\circ}$ ya que no sabemos si los alumnos emplean de manera espontánea los fenómenos de aproximación intuitiva, o si lo hacen por contaminación de la respuesta del alumno ficticio.

-No tenemos argumentos para afirmar que los alumnos manejen los fenómenos de retroalimentación en sucesiones, ya que no manejan la idea de entorno, palabra empleada en el enunciado de la cuestión como elemento de los fenómenos de retroalimentación.

-Creemos haber logrado el objetivo general $3^{\circ}$, ya que hemos observado un mayor porcentaje de éxito en la cuestión en la que se empleaba el sistema de representación gráfico, estableciéndose así diferencias entre éste y los sistemas de representación verbal y tabular.

-Creemos haber logrado el objetivo general $4^{\circ}$, ya que hemos detectado que al dar una posible respuesta a la cuestión presentada (respuesta de un alumno ficticio), ésta se emplea en las respuestas de los alumnos.

\section{Anexo 5 Cuestionario}

El cuestionario consta de 5 páginas. En las dos primeras se incluyen instrucciones y en las otras tres, las preguntas propiamente dichas, una por página. Cada pregunta se mostró en un recuadro.

Página $1^{a}$ del cuestionario

INSTRUCCIONES PARA LA REALIZACIÓN DEL CUESTIONARIO:

1) El cuestionario tiene una duración de 30 minutos y se realiza por cada alumno de manera individual. 
2) En cada pregunta se da una justificación de un alumno y se pide une aclaración. Como aclaración, por favor, no emplees las mismas palabras que ese alumno. Es decir, cuando contestes sobre el límite de la sucesión presentada, esperamos que justifiques dicha afirmación de manera razonada, y utilizando tus propias palabras.

3) Para justificar tus respuestas, puedes emplear el modo que consideres más conveniente.

4) Por favor, como esto no es un examen, contesta todas las cuestiones, sin dejar en blanco ninguna de ellas.

5) Si deseas conocer algún término de la sucesión que no aparece en la cuestión presentada, pregunta al profesor, el cual te lo suministrará.

6) El cuestionario solamente puede ser realizado por alumnos de $1^{\circ}$ y $2^{\circ}$ de bachillerato, de ciencias sociales, de ciencias de la naturaleza y de la salud y bachillerato tecnológico.

7) Los datos de clasificación: nombre, curso, centro, edad, etc, son recogidos para observar si existen diferencias importantes entre el tratamiento del concepto de límite en los diferentes cursos en los que se imparte. Asimismo recogemos la asignatura que los alumnos estudian para observar si también existe diferente tratamiento entre un bachillerato y otro.

8) Los datos personales, así como las respuestas obtenidas serán tratadas de manera confidencial. En nuestro estudio no citaremos el nombre de ningún alumno, manteniendo de esta manera su anonimato. Además, las respuestas individuales de los alumnos se utilizarán para describir las características del grupo. Gracias por la colaboración.

Página $2^{a}$ del cuestionario

Cuestionario

Nombre:

Curso:

Centro:

Edad:

Repite curso:

Usáis libro de texto en clase:

Nota de matemáticas en el último curso

¿Cuál?

Rodea el nombre de la asignatura de matemáticas que cursas:

- Matemáticas I.

- Matemáticas II.

- Matemáticas aplicadas a las ciencias Sociales I.

- Matemáticas aplicadas a las ciencias Sociales II.

Páginas $3^{a}$ del cuestionario. Cuestión $n^{o} 1$.

1) Se representa gráficamente una sucesión An. El profesor pregunta a la clase: ¿Tiene límite la sucesión? Un alumno responde: "El limite es 3 porque a medida que $\mathrm{n}$ crece los valores de la sucesión se van acercando cada vez más a 3”. ¿Estás de acuerdo con el? Justifica tu respuesta de manera razonada.

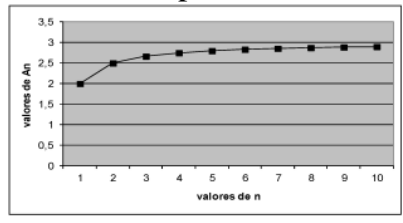

Página $4^{a}$ del cuestionario. Cuestión $n^{o} 2$.

2) Dada la siguiente sucesión:

\begin{tabular}{|l|l|l|l|l|l|l|l|}
\hline $\mathrm{n}$ & 1 & 2 & 10 & 1000 & 1000000 & 1000000000 & $\ldots \ldots \ldots \ldots \ldots \ldots$. \\
\hline $\mathrm{a}_{\mathrm{n}}$ & 0,571428 & 0,7 & 1,24 & 1,496758 & 1,49999675 & 1,49999999 & $\ldots \ldots \ldots \ldots \ldots \ldots .$. \\
\hline
\end{tabular}

El profesor pregunta a la clase: ¿Tiene límite la sucesión $a_{n}$ ? Un alumno responde: "El límite es 1,5 porque a medida que avanzo en la sucesión los valores de $a_{n}$ se aproximan más a 1,5”. ¿Estás de acuerdo con él? Justifica tu respuesta de manera razonada.

Página $5^{a}$ del cuestionario. Cuestión $n^{o} 3$.

3) La sucesión $a_{n}$ cumple: su primer término vale 2.9, su segundo término vale 2.99, su tercer término vale 2.999, su cuarto término 2.9999, su quinto término 2.99999, su sexto término 2.999999, su séptimo término 2.9999999, su octavo término 2.99999999 y así sucesivamente.

a) Un alumno afirma: "Esta sucesión tiene límite 3, porque a medida que avanza n su valor va acercándose cada vez más a 3" ¿Estás de acuerdo con él? Justifica tu respuesta de manera razonada.

b) Otro alumno afirma: "La sucesión tiene límite 3 porque la distancia entre los términos de la sucesión y 3, puede hacerse tan pequeña como se desee, a partir de un término convenientemente elegido" ¿Estás de acuerdo con él? Justifica tu respuesta de manera razonada. 